\title{
Constraints on the $\omega \pi$ form factor from analyticity and unitarity
}

\section{B. Ananthanarayan*}

Centre for High Energy Physics, Indian Institute of Science, Bangalore 560 012, India

E-mail: anant@cts.iisc.ernet.in

\section{Irinel Caprini}

Horia Hulubei National Institute for Physics and Nuclear Engineering, P.O.B. MG-6, 077125

Bucharest-Magurele, Romania

E-mail: caprini@theory.nipne.ro

\section{Bastian Kubis}

Helmholtz-Institut für Strahlen- und Kernphysik (Theorie) and Bethe Center for Theoretical Physics, Universität Bonn, D-53115 Bonn, Germany

E-mail: kubis@hiskp.uni-bonn.de

In this talk we review our recent work on the electromagnetic $\omega \pi$ form factor in a modelindependent framework known as the method of unitarity bounds. Our study was motivated by the discrepancies noted recently between the theoretical calculations of the form factor based on dispersion relations and certain experimental data measured from the decay $\omega \rightarrow \pi^{0} \gamma^{*}$. We have applied a modified dispersive formalism, which uses as input the discontinuity of the $\omega \pi$ form factor calculated by unitarity below the $\omega \pi$ threshold and an integral constraint on the square of its modulus above this threshold. The latter constraint was obtained by exploiting unitarity and the positivity of the spectral function of a QCD correlator, computed on the spacelike axis by operator product expansion and perturbative QCD. From these conditions we derived upper and lower bounds on the modulus of the $\omega \pi$ form factor in the region below the $\omega \pi$ threshold. The results confirm the existence of a disagreement between dispersion theory and experimental data on the $\omega \pi$ form factor around $0.6 \mathrm{GeV}$.

The 8th International Workshop on Chiral Dynamics, CD2015

29 June 2015 - 03 July 2015

Pisa, Italy

\footnotetext{
* Speaker.
} 


\section{Introduction}

There is renewed interest in the transition form factors of light mesons [1], partly because the pseudoscalar $\left(\pi^{0}, \eta, \eta^{\prime}\right)$ pole terms constitute some of the most important contributions to hadronic light-by-light scattering, which enters the standard model prediction for the muon's anomalous magnetic moment (see [2] and references therein). Some of them can be measured in decays such as $\omega \rightarrow \pi^{0} \ell^{+} \ell^{-}$or $\phi \rightarrow \eta \ell^{+} \ell^{-}$.

The present contribution is based on the work reported in the recent publications [3, 4]. The motivation of these studies was the fact that recent dispersive treatments $[5,6]$ of the $\omega \pi$ electromagnetic form factor $f_{\omega \pi}(t)$ are in disagreement with experimental data in the region around $0.6 \mathrm{GeV}[7,8,9]$, which show strong deviations from even approximate vector-meson-dominance behavior [10] and descriptions based on effective field theory [11, 12]. The dispersive method uses unitarity, which allows one to express the discontinuity of the form factor in terms of the $\mathrm{P}$ partial wave of the process $\pi \pi \rightarrow \omega \pi[13,6]$ and the pion electromagnetic form factor, quantities determined with precision. Strictly speaking, this relation is valid only in the elastic region, $4 m_{\pi}^{2} \leq t<16 m_{\pi}^{2}$. Phenomenologically, it is known that the elastic regime approximately extends up to $\omega \pi$ threshold, $t_{+}=\left(m_{\omega}+m_{\pi}\right)^{2}$, above which the inelasticity in the P-wave pion-pion scattering is assumed to be dominated by the $\omega \pi$ intermediate state [13]. However, due to the lack of information on the discontinuity in the inelastic region, in previous studies it was assumed that elastic unitarity is valid also at higher energies in the evaluation of the dispersion integral. This assumption can affect the precision of the theoretical treatment.

In order to avoid the assumption above we have exploited alternative information on the form factor above $t_{+}$. In Ref. [3] we have used a method proposed originally by Okubo [14] (before the advent of QCD), which leads to bounds on form factors by exploiting the analyticity and the positivity properties of the spectral function of a suitable current-current correlator. The method has come to be known as method of unitarity bounds, and has been resuscitated in the QCD era (for a review and more references see $[15,16]$ ). In the present case we use a dispersion relation for the polarization function of two isovector vector currents, calculated by operator product expansion (OPE) in the Euclidean region, and exploit unitarity for the spectral function. Including $\pi \pi$ and $\omega \pi$ states in the unitarity sum, we derived an upper bound for an integral on the modulus squared of the $\omega \pi$ form factor along the cut from $t_{+}$to infinity [3]. As discussed in the subsequent work [4], instead of this theoretical condition derived from analyticity and unitarity, one can constrain the modulus of the $\omega \pi$ form factor above $t_{+}$using experimental information from the reaction $e^{+} e^{-} \rightarrow$ $\omega \pi^{0}$, reported in $[17,18,19]$.

From the constraint on the modulus along the cut above $t_{+}$and the known discontinuity in the region $4 m_{\pi}^{2} \leq t<t_{+}$, it is possible to derive bounds on the values of the form factor in the complex $t$-plane and on the real axis below $t_{+}$. For deriving these bounds, we have adapted the original method of unitarity bounds, making it suitable for the information available for the $\omega \pi$ form factor, namely its discontinuity across the cut below the inelastic threshold. The corresponding optimization problem was solved by analytic techniques already applied to scattering amplitudes [20,21]. We also had to take into account the fact that, unlike most form factors studied up to now, the $\omega \pi$ form factor $f_{\omega \pi}(t)$ is not a real analytic function, i.e. it does not satisfy the condition $f_{\omega \pi}\left(t^{*}\right)=\left(f_{\omega \pi}(t)\right)^{*}$. We have therefore made the proper generalization of the formalism to the 
case of analytic functions that are not of real type, a feature worth emphasizing.

\section{Standard dispersive treatment of $f_{\omega \pi}(t)$}

We use the definition from [5], where the form factor $f_{\omega \pi}(t)$ is defined from the matrix element

$$
\left\langle\omega\left(p_{a}, \lambda\right) \pi\left(p_{b}\right)\left|j_{\mu}(0)\right| 0\right\rangle=i \varepsilon_{\mu \tau \rho \sigma} \varepsilon^{\tau *}\left(p_{a}, \lambda\right) p_{b}^{\rho} q^{\sigma} f_{\omega \pi}(t)
$$

where $j_{\mu}$ is the isovector part of the electromagnetic current, $\lambda$ denotes the $\omega$ polarization, and we defined $q=p_{a}+p_{b}$ and $t=q^{2}$. The form factor $f_{\omega \pi}(t)$ has dimension $\mathrm{GeV}^{-1}$.

Unitarity implies that $f_{\omega \pi}(t)$ has a cut along the real axis for $t \geq 4 m_{\pi}^{2}$. Using the conventions of [5], the discontinuity of $f_{\omega \pi}(t)$ across the cut in the elastic approximation is given by

$$
\operatorname{disc} f_{\omega \pi}(t)=\frac{i q_{\pi \pi}^{3}(t)}{6 \pi \sqrt{t}} F_{\pi}^{*}(t) f_{1}(t) \theta\left(t-4 m_{\pi}^{2}\right),
$$

where $q_{\pi \pi}(t)=\sqrt{t / 4-m_{\pi}^{2}}, F_{\pi}(t)$ is the pion electromagnetic form factor, and $f_{1}(t)$ the P partialwave amplitude of the scattering process

$$
\pi^{+}\left(q_{1}\right) \pi^{-}\left(q_{2}\right) \rightarrow \omega\left(p_{a}, \lambda\right) \pi^{0}\left(p_{b}\right)
$$

We have evaluated the discontinuity with input from two different dispersive treatments of the $\omega \pi$ form factor. In the first treatment, reported in the older paper [22], the pion form factor $F_{\pi}(t)$ was described by a Gounaris-Sakurai representation, while in the recent work [5] this form factor has been reconstructed from an Omnès representation [23], using as input the pion-pion phase shift $\delta_{1}^{1}(t)$ calculated from Roy equations [24, 25]. The differences between the two representations of the pion form factor are very small and have a negligible influence on the results.

On the other hand, the calculation of the partial wave $f_{1}(t)$ in the above two references is quite different. In Ref. [22], $f_{1}(t)$ was calculated by the $N / D$ method, with the left-hand cut approximated by $\rho$-exchange. In this model, the phase of $f_{1}(t)$ exactly compensates the phase of $F_{\pi}^{*}(t)$ in the discontinuity (2.2), and as a consequence the form factor $f_{\omega \pi}(t)$ is a real analytic function. However, as discussed in [5], once rescattering effects are taken into account, the phase of the partial wave $f_{1}(t)$ no longer coincides with the $\pi \pi$ P-wave phase shift as would be implied by the naive application of Watson's theorem [26]. More precisely, since in the region where the decay

$$
\omega\left(p_{a}, \lambda\right) \rightarrow \pi^{+}\left(q_{1}\right) \pi^{-}\left(q_{2}\right) \pi^{0}\left(-p_{b}\right)
$$

is allowed, the rescattering between the final pions including three-pion cuts is possible, the discontinuity (2.2) is not purely imaginary and therefore the $\omega \pi$ form factor is not a real analytic function. Rescattering effects are properly taken into account in the calculation of the partial wave $f_{1}$ performed in [5, 13], using Khuri-Treiman techniques [27].

The expression (2.2) is valid in the region $4 m_{\pi}^{2} \leq t<t_{+}$, since above the $\omega \pi$ threshold other intermediate states contribute in the unitarity sum. By neglecting these contributions, the form factor was obtained from a once-subtracted dispersion relation [5, 22]

$$
f_{\omega \pi}(t)=f_{\omega \pi}(0)+\frac{t}{2 \pi i} \int_{4 m_{\pi}^{2}}^{\infty} \frac{\operatorname{disc} f_{\omega \pi}\left(t^{\prime}\right)}{t^{\prime}\left(t^{\prime}-t\right)} d t^{\prime} .
$$


The quantity $\left|f_{\omega \pi}(0)\right|$ is known experimentally from the $\omega \rightarrow \pi^{0} \gamma$ decay rate. The updated value is [28]

$$
\left|f_{\omega \pi}(0)\right|=(2.30 \pm 0.04) \mathrm{GeV}^{-1} .
$$

The recent analysis performed in [5], based on the dispersion relation (2.5), leads to results that are inconsistent with some experimental data around $\sqrt{t} \approx 0.6 \mathrm{GeV}[7,8,9]$. As discussed above, one questionable point of the theoretical analysis is the extension of the elastic unitarity relation (2.2) above the (effective) threshold $t_{+}=\left(m_{\omega}+m_{\pi}\right)^{2}$ of multiparticle production. In the next section we shall show how some information on the modulus of the form factor $f_{\omega \pi}(t)$ for $t>t_{+}$can be derived from independent sources.

\section{Consequences of perturbative QCD, analyticity and unitarity}

We begin with the polarization tensor

$$
\Pi^{\mu v}(q)=\int d x e^{i q x}\left\langle 0\left|T\left[j^{\mu}(x) j^{v}(0)\right]\right| 0\right\rangle=\left(q^{\mu} q^{v}-g^{\mu v} q^{2}\right) \Pi(t), \quad t=q^{2},
$$

where $j_{\mu}$ is the isovector part of the electromagnetic current. The first derivative $\Pi^{\prime}(t)$ of the QCD vacuum polarization amplitude $\Pi(t)$ satisfies the dispersion relation

$$
\Pi^{\prime}(t)=\frac{1}{\pi} \int_{0}^{\infty} \frac{\operatorname{Im} \Pi\left(t^{\prime}+i \varepsilon\right)}{\left(t^{\prime}-t\right)^{2}} d t^{\prime}
$$

with the spectral function given by the unitarity relation

$$
\left(q^{\mu} q^{v}-g^{\mu v} q^{2}\right) \operatorname{Im} \Pi(t+i \varepsilon)=\frac{1}{2} \sum_{\Gamma} \int d \rho_{\Gamma}(2 \pi)^{4} \delta^{(4)}\left(q-p_{\Gamma}\right)\left\langle 0\left|j^{\mu}(0)\right| \Gamma\right\rangle\left\langle\Gamma\left|j^{v}(0)^{\dagger}\right| 0\right\rangle .
$$

Keeping the $\pi \pi$ and $\omega \pi$ intermediate states explicitly (which should dominate the isovector spectral function at low energies), carrying out the two-body phase space integrals and using the positivity of the spectral function, we obtain the inequality

$$
\Pi^{\prime}(t) \geq \int_{4 m_{\pi}^{2}}^{\infty} w_{\pi}\left(t^{\prime}, t\right)\left|F_{\pi}\left(t^{\prime}\right)\right|^{2} d t^{\prime}+\int_{t_{+}}^{\infty} w_{\omega \pi}\left(t^{\prime}, t\right)\left|f_{\omega \pi}\left(t^{\prime}\right)\right|^{2} d t^{\prime}
$$

where

$$
\begin{aligned}
w_{\pi}\left(t^{\prime}, t\right) & =\frac{1}{48 \pi^{2}} \frac{1}{\left(t^{\prime}-t\right)^{2}}\left(1-\frac{4 m_{\pi}^{2}}{t^{\prime}}\right)^{3 / 2} \\
w_{\omega \pi}\left(t^{\prime}, t\right) & =\frac{1}{192 \pi^{2}} \frac{t^{\prime}}{\left(t^{\prime}-t\right)^{2}}\left(1-\frac{t_{-}}{t^{\prime}}\right)^{3 / 2}\left(1-\frac{t_{+}}{t^{\prime}}\right)^{3 / 2}
\end{aligned}
$$

and we denoted $t_{ \pm}=\left(m_{\omega} \pm m_{\pi}\right)^{2}$. It is convenient to write (3.4) as an integral constraint on the square of the modulus of the $\omega \pi$ form factor as

$$
\int_{t_{+}}^{\infty} w_{\omega \pi}\left(t^{\prime}, t\right)\left|f_{\omega \pi}\left(t^{\prime}\right)\right|^{2} d t^{\prime} \leq I(t)
$$

where

$$
I(t)=\Pi^{\prime}(t)-\int_{4 m_{\pi}^{2}}^{\infty} w_{\pi}\left(t^{\prime}, t\right)\left|F_{\pi}\left(t^{\prime}\right)\right|^{2} d t^{\prime}
$$


This quantity can be evaluated for spacelike values $t \equiv-Q^{2}<0$ using OPE and perturbative QCD for the correlator $\Pi^{\prime}(t)$, and the rich information available on the modulus of the pion form factor. The value of $Q^{2}$ should be taken large enough such as to ensure the validity of the OPE, and in the same time lead to sufficiently strong bounds. As discussed in [29], a reasonable choice satisfying these requirements is $Q^{2}=2 \mathrm{GeV}^{2}$. Perturbative QCD to four loops (see [30] and references therein) gives:

$$
\Pi_{\text {pert }}^{\prime}\left(-Q^{2}\right)=\frac{1}{8 \pi^{2} Q^{2}}\left(1+0.318 \alpha_{s}+0.166 \alpha_{s}^{2}+0.205 \alpha_{s}^{3}+0.504 \alpha_{s}^{4}\right),
$$

where $\alpha_{s}$ is the strong coupling at $Q^{2}=2 \mathrm{GeV}^{2}$. Using as input the value $\alpha_{s}\left(m_{\tau}^{2}\right)=0.320 \pm 0.020$, which covers most of the recent determinations from hadronic $\tau$ decays [28] and the coupling's running we obtain $\alpha_{s}=0.357 \pm 0.025$. This yields for $\Pi_{\text {pert }}^{\prime}$ the central value $0.0073 \mathrm{GeV}^{-2}$ with an error of about $1.3 \%$. We also checked that a higher-order term added in (3.8), taken as $0.925 \alpha_{s}^{5}$ according to [31], changes $\Pi_{\text {pert }}^{\prime}$ by about $1.2 \%$. The power corrections in the OPE, obtained from [32], bring a small contribution. For instance, using the standard value $\left\langle\alpha_{s} G^{2}\right\rangle / \pi=0.012 \mathrm{GeV}^{4}$ for the gluon condensate given in [33], we obtain for its contribution the value $0.0001 \mathrm{GeV}^{-2}$. This leads to $\Pi^{\prime}\left(-2 \mathrm{GeV}^{2}\right)=(0.0074 \pm 0.0001) \mathrm{GeV}^{-2}$, where the uncertainty includes the effects of the $\alpha_{s}$ uncertainty and the truncation error, added quadratically.

The integral involving the pion electromagnetic form factor can be calculated using in the lowenergy region BaBar experimental data [34] and the bounds on $\left|F_{\pi}(t)\right|$ derived in [35], along with data obtained by BaBar up to $3 \mathrm{GeV}$ [34] and a smooth transition to the $1 / t$ decrease predicted by QCD (for details see [35, 36]). This gives for the integral appearing in (3.7) the value $(0.0033 \pm$ $0.0001) \mathrm{GeV}^{-2}$, which leads to

$$
I \equiv I\left(-2 \mathrm{GeV}^{2}\right)=(0.0041 \pm 0.0002) \mathrm{GeV}^{-2} .
$$

From the inequality (3.6) and the discontinuity (2.2) adopted in the elastic region of validity $t<t_{+}$, we shall obtain bounds on the form factor at points below $t_{+}$. The mathematical technique will be briefly described in the next section.

\section{Bounds on $\left|f_{\omega \pi}(t)\right|$ below the $\omega \pi$ threshold}

In order to express the input conditions in a canonical form, the first step is to map the $t$ plane cut along $t \geq t_{+}$onto the unit disk $|z|<1$ in the $z \equiv \tilde{z}(t)$ plane, by using a suitable conformal mapping. In particular we shall use the mapping

$$
\tilde{z}(t)=\frac{1-\sqrt{1-t / t_{+}}}{1+\sqrt{1-t / t_{+}}},
$$

such that the $\tilde{z}(0)=0$. In the $z$-plane the elastic region $4 m_{\pi}^{2} \leq t<t_{+}$becomes the segment $x_{\pi} \leq$ $x<1$ of the real axis, where $x_{\pi}=\tilde{z}\left(4 m_{\pi}^{2}\right)$, and the upper (lower) edges of the cut $t>t_{+}$become the upper (lower) semicircles.

Further, we shall construct an outer function $C(z)$, i.e. a function analytic and without zeros in $|z|<1$, its modulus on $|z|=1$ being equal to $\sqrt{w_{\omega \pi}\left(\tilde{t}(z),-Q^{2}\right)|d \tilde{t}(z) / d z|}$, where $\tilde{t}(z)$ is the inverse 
of (4.1). The construction of the outer functions is explained in [37] (see also the review [15]). Using the expression (3.5) of $w_{\omega \pi}\left(t,-Q^{2}\right)$, we obtain for $C(z)$ the exact analytic expression

$$
C(z)=\frac{(1-z)^{2}(1+z)^{-1 / 2}}{16 \sqrt{6} \pi} \frac{\left(1+\tilde{z}\left(-Q^{2}\right)\right)^{2}\left(1-z \tilde{z}\left(t_{-}\right)\right)^{3 / 2}}{\left(1-z \tilde{z}\left(-Q^{2}\right)\right)^{2}\left(1+\tilde{z}\left(t_{-}\right)\right)^{3 / 2}} .
$$

Then the inequality (3.6), written in terms of the new function $h(z)$ defined as

$$
h(z)=C(z) f_{\omega \pi}(\tilde{t}(z)) .
$$

is written in the equivalent form

$$
\frac{1}{2 \pi} \int_{0}^{2 \pi} d \theta\left|h\left(e^{i \theta}\right)\right|^{2} \leq I
$$

where $\theta=\arg z$.

Since $C(z)$ is real analytic in $|z|<1, C(x)$ is real for $x_{\pi} \leq x<1$, and from the definition (4.3) it follows that we can write

$$
\operatorname{disc} h(x) \equiv \Delta(x)=C(x) \operatorname{disc} f_{\omega \pi}(\tilde{t}(x)),
$$

where the discontinuity of the form factor is obtained from (2.2). The function $h(z)$ can be expressed in terms of its discontinuity as

$$
h(z)=\frac{1}{2 \pi i} \int_{x_{\pi}}^{1} \frac{\Delta(x)}{x-z} d x+g(z),
$$

where the function $g(z)$ is analytic in $|z|<1$, as its discontinuity across the real axis vanishes:

$$
\operatorname{disc} g(x)=0, \quad-1<x<1 .
$$

Since we consider in general form factors that are not real analytic, the function $g(z)$ is analytic, but its values on the real axis may be complex.

We now express the available information on the form factor as a number of constraints on the function $g$. By inserting (4.6) in (4.4) we obtain the condition

$$
\frac{1}{2 \pi} \int_{0}^{2 \pi} d \theta\left|\frac{1}{2 \pi i} \int_{x_{\pi}}^{1} \frac{\Delta(x)}{x-e^{i \theta}} d x+g\left(e^{i \theta}\right)\right|^{2} \leq I,
$$

while from (2.6) it follows that $g(0)$ has the value

$$
g(0)=f_{\omega \pi}(0) C(0)-\frac{1}{2 \pi i} \int_{x_{\pi}}^{1} \frac{\Delta(x)}{x} .
$$

The problem is to find the maximal allowed range of $|g(z)|$ at an arbitrary given point $z=\tilde{z}(t)$ in the interval $\left(x_{\pi}, 1\right)$, for functions $g(z)$ analytic in $|z|<1$ and subject both to the boundary condition (4.8) and the additional constraint (4.9). Using the method of Lagrange multipliers, the following upper and lower bounds on $\left|f_{\omega \pi}(t)\right|$ for $t \in\left(4 m_{\pi}^{2}, t_{+}\right)$were obtained and presented in [3]:

$$
\begin{aligned}
& \left|f_{\omega \pi}(t)\right| \leq \frac{\left|g(0)+\frac{1}{2 \pi i} \int_{x_{\pi}}^{1} \frac{\Delta(x)}{x-z} d x\right|+\frac{z I^{\prime}}{\sqrt{1-z^{2}}}}{C(z)}, \\
& \left|f_{\omega \pi}(t)\right| \geq \frac{\left|g(0)+\frac{1}{2 \pi i} \int_{x_{\pi}}^{1} \frac{\Delta(x)}{x-z} d x\right|-\frac{z I^{\prime}}{\sqrt{1-z^{2}}}}{C(z)},
\end{aligned}
$$


where $z=\tilde{z}(t)$ and

$$
I^{\prime}=\left[I-\frac{1}{4 \pi^{2}} \int_{x_{\pi}}^{1} \int_{x_{\pi}}^{1} \frac{\Delta(x) \Delta^{*}(y)}{1-x y} d x d y-|g(0)|^{2}\right]^{1 / 2} .
$$

We have taken into account the fact that $C(z)$ calculated from (4.2) is positive for $-1<z<1$. Using the value of $g(0)$ from (4.9) and $I^{\prime}$ from (4.11), with $\Delta(x)$ defined in (4.5), we have all the ingredients to evaluate (4.10) numerically at an arbitrary point $4 m_{\pi}^{2}<t<t_{+}$.

In the particular case of real analytic functions, optimal upper and lower bounds on the real part of the function in the range $4 m_{\pi}^{2}<t<t_{+}$can be derived using the methods developed in [20,21]. In the present case the bounds are [3]:

$$
\begin{aligned}
& \operatorname{Re} f_{\omega \pi}(t) \leq \frac{g(0)+\frac{P}{\pi} \int_{x_{\pi}}^{1} \frac{\sigma(x)}{x-z} d x+\frac{z I^{\prime}}{\sqrt{1-z^{2}}}}{C(z)}, \\
& \operatorname{Re} f_{\omega \pi}(t) \geq \frac{g(0)+\frac{P}{\pi} \int_{x_{\pi}}^{1} \frac{\sigma(x)}{x-z} d x-\frac{z I^{\prime}}{\sqrt{1-z^{2}}}}{C(z)},
\end{aligned}
$$

where $P$ denotes the Principal Value and $\sigma(x)=C(x) \operatorname{Im} f_{\omega \pi}(\tilde{t}(x))$.

\section{Results}

In the calculation of the bounds we have employed the discontinuity of the $\omega \pi$ form factor in the range $\left(4 m_{\pi}^{2}, t_{+}\right)$from two different analyses: the recent dispersive treatment reported in [5] and the older work [22]. For the quantity $I$ we used the estimate given in (3.9). It turns out that the value of the form factor at $t=0$ plays a significant role in producing stringent constraints. Although in principle $f_{\omega \pi}(0)$ can be complex, we have assumed that it only has a small phase which can be neglected [5]. The upper and lower bounds given below were obtained using as input the central value $f_{\omega \pi}(0)=2.30 \mathrm{GeV}^{-1}$.

We have checked that the bounds are quite stable with respect to the variation of the input: by varying $f_{\omega \pi}(0)$ inside the error quoted in (2.6), the upper bounds in the region of interest are changed by at most $2.5 \%$. Also, the uncertainty of the quantity $I$ quoted in (3.9) affects the bounds by at most $2 \%$. As the experimental errors are currently rather in the 10-20\% range, we refrain from displaying these small variations in the bounds graphically and only discuss the central results.

Our results are presented in Fig. 1, which shows upper and lower bounds on the modulus squared (normalized to its value at $t=0$ ) in region $t<t_{-}$accessible experimentally in $\omega \rightarrow$ $\pi^{0} \mu^{+} \mu^{-}$. For the input from [5], when the form factor is not a real analytic function, the bounds on $\left|f_{\omega \pi}(t)\right|$ were calculated using (4.10). For the input from [22], where rescattering effects are neglected and the form factor is real analytic, we used the optimal bounds (4.12) on the real part, and combined them with the knowledge of the imaginary part to obtain bounds on the modulus. For comparison, we also show the result of the dispersive calculation performed in [5], as well as several experimental data from [7, 8, 9]. Fig. 1 shows that, although the allowed ranges for the ratio $\left|f_{\omega \pi}(t) / f_{\omega \pi}(0)\right|^{2}$ are rather large, some of the experimental data points are excluded, which is the essential point brought out by our analysis. 


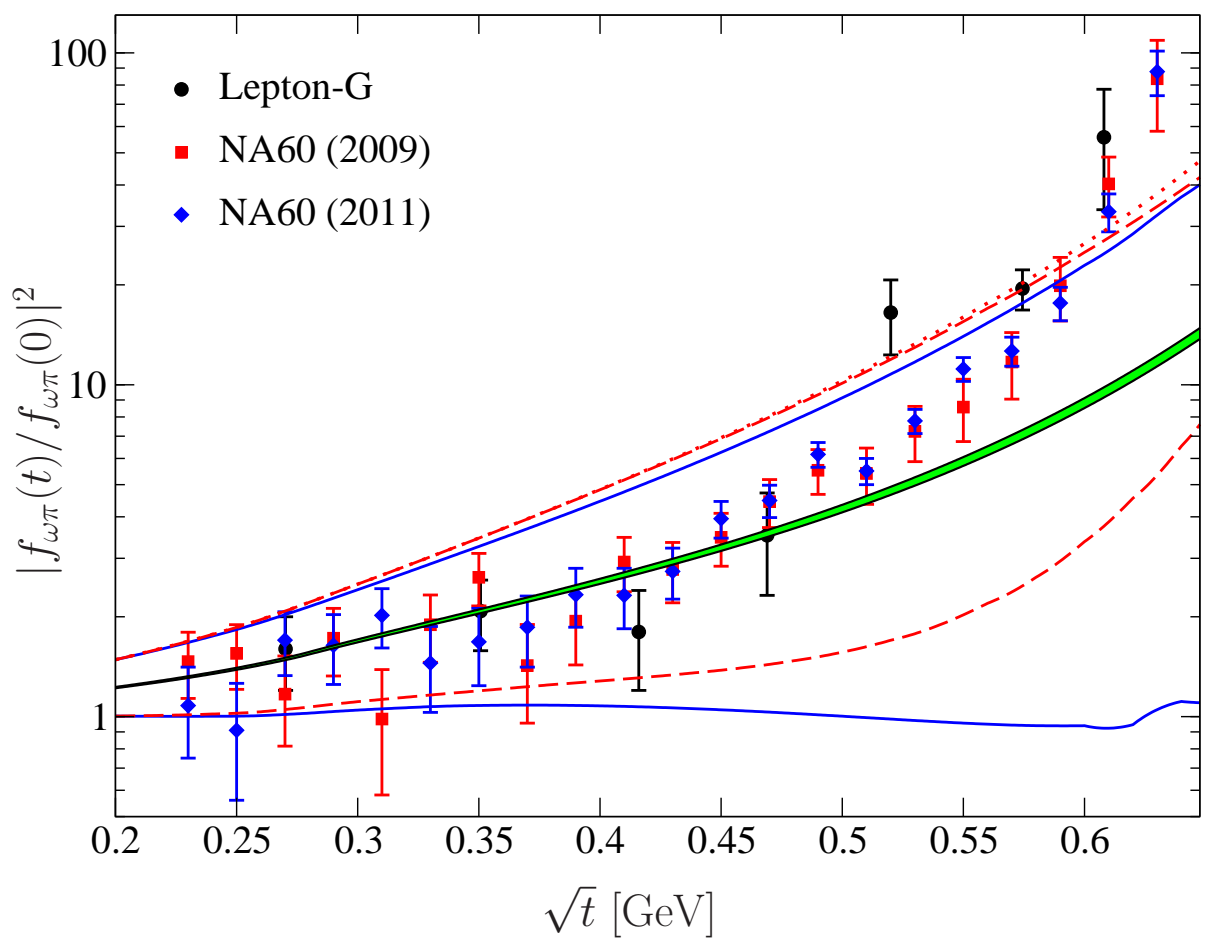

Figure 1: Upper and lower bounds on $\left|f_{\omega \pi}(t) / f_{\omega \pi}(0)\right|^{2}$ from our formalism [3] with the input from [5] shown in blue and bounds with input from [22] shown in red. Also shown are the experimental data from Lepton-G [7], NA60 (2009) [8], and NA60 (2011) [9]. The green band is the result of the dispersive calculation performed in [5].

As mentioned in the introduction, instead of the theoretical constraint (3.6) one can use as input experimental information on the modulus $\left|f_{\omega \pi}(t)\right|$ for $t>t_{+}$measured from the reaction $e^{+} e^{-} \rightarrow$ $\omega \pi^{0}$. This approach was considered in [4], where also an improved version of the $N / D$ model of the partial wave $f_{1}(t)$ given in [22] was used as input. In Fig. 2, we show the allowed values of $\left|f_{\omega \pi}(t) / f_{\omega \pi}(0)\right|^{2}$ defined by the upper and lower bounds calculated in [4] in the whole region $t<t_{+}$. A comparison with Fig. 1 shows that the bounds obtained with experimental information are much more stringent than the "unitarity bounds" derived in [3], using only theoretical constraints above $t_{+}$. The important and persistent message is that some experimental data around $0.6 \mathrm{GeV}$ are in conflict with these predictions, being situated well above the upper bounds.

\section{Discussion and conclusions}

The present study was motivated by the discrepancies noticed recently between the theoretical calculation of the $\omega \pi$ form factor by a dispersion relation and some of the data reported in [7, 8, 9]. Our aim was to avoid the assumptions made on the discontinuity of the form factor above the threshold $t_{+}$, where the elastic unitarity (2.2) is no longer valid. To this end we have resorted to the formalism of unitarity bounds. The central point of the formalism is the derivation of an integral condition on the modulus squared of the form factor from $t_{+}$to infinity, which can be calculated using OPE and perturbative QCD in the Euclidean region for a suitable correlator, together with 


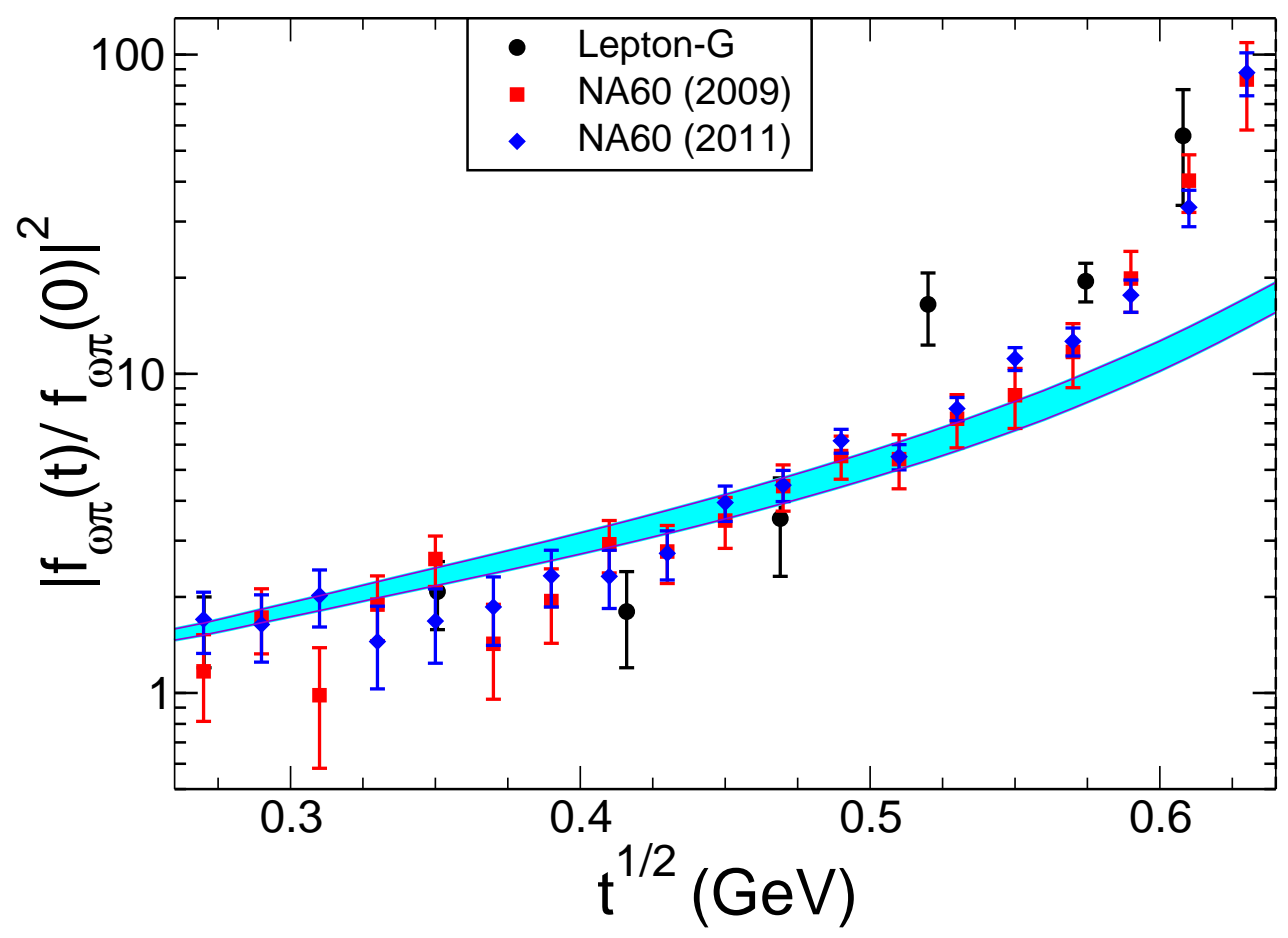

Figure 2: Cyan band: allowed values of $\left|f_{\omega \pi}(t) / f_{\omega \pi}(0)\right|^{2}$ in the region $t<t_{+}$defined by the upper and lower bounds derived in Ref. [4], compared with experimental data from Lepton-G [7], NA60 (2009) [8], and NA60 (20 11) [9].

unitarity and positivity of the spectral function. In the present case it was convenient to consider the tensor (3.1) of two isovector currents.

In this work we have considered a modified version of the standard formalism, suitable for including the information available on the $\omega \pi$ form factor, namely the discontinuity (2.2) known in the elastic region. This is a generalization of the formalism of unitarity bounds, considered for the first time in our work [3]. The framework is not specific to the $\omega \pi$ form factor and could be easily adapted to the analysis of other form factors. The derivation uses the maximization of the modulus of a difference of complex numbers in the last step, which implies that strictly speaking the bounds (4.10) are not optimal.

The numerical results show that several experimental data around $0.6 \mathrm{GeV}$ are situated above the upper bounds derived in $[3,4]$. Having in view the model-independent treatment of the region above the inelastic threshold $t_{+}$adopted in these analyses, the disagreement signals possible problems with the experimental data.

\section{References}

[1] E. Czerwiński et al., arXiv:1207.6556 [hep-ph].

[2] F. Jegerlehner and A. Nyffeler, Phys. Rept. 477 (2009) 1 [arXiv:0902.3360 [hep-ph]].

[3] B. Ananthanarayan, I. Caprini and B. Kubis, Eur. Phys. J. C 74 (2014) 12, 3209 [arXiv:1410.6276 [hep-ph]]. 
[4] I. Caprini, Phys. Rev. D 92 (2015) 014014 [arXiv:1505.05282 [hep-ph]].

[5] S. P. Schneider, B. Kubis and F. Niecknig, Phys. Rev. D 86 (2012) 054013 [arXiv:1206.3098 [hep-ph]].

[6] I. V. Danilkin, C. Fernández-Ramírez, P. Guo, V. Mathieu, D. Schott, M. Shi and A. P. Szczepaniak, Phys. Rev. D 91 (2015) 9, 094029 [arXiv:1409.7708 [hep-ph]].

[7] R. I. Dzhelyadin et al., Phys. Lett. B 102 (1981) 296 [JETP Lett. 33 (1981) 228].

[8] R. Arnaldi et al. [NA60 Collaboration], Phys. Lett. B 677 (2009) 260 [arXiv:0902.2547 [hep-ph]].

[9] G. Usai [NA60 Collaboration], Nucl. Phys. A 855 (2011) 189.

[10] L. G. Landsberg, Phys. Rept. 128 (1985) 301.

[11] C. Terschlüsen and S. Leupold, Phys. Lett. B 691 (2010) 191 [arXiv:1003.1030 [hep-ph]].

[12] C. Terschlüsen, S. Leupold and M. F. M. Lutz, Eur. Phys. J. A 48 (2012) 190 [arXiv:1204.4125 [hep-ph]].

[13] F. Niecknig, B. Kubis and S. P. Schneider, Eur. Phys. J. C 72 (2012) 2014 [arXiv:1203.2501 [hep-ph]].

[14] S. Okubo, Phys. Rev. D 3 (1971) 2807; Phys. Rev. D 4 (1971) 725.

[15] G. Abbas, B. Ananthanarayan, I. Caprini, I. Sentitemsu Imsong and S. Ramanan, Eur. Phys. J. A 45 (2010) 389 [arXiv:1004.4257 [hep-ph]].

[16] B. Ananthanarayan and I. Caprini, J. Phys. Conf. Ser. 374 (2012) 012011 [arXiv:1202.5391 [hep-ph]].

[17] M. N. Achasov et al. [SND Collaboration], Phys. Lett. B 486 (2000) 29 [hep-ex/0005032].

[18] R. R. Akhmetshin et al. [CMD-2 Collaboration], Phys. Lett. B 562 (2003) 173 [hep-ex/0304009].

[19] F. Ambrosino et al. [KLOE Collaboration], Phys. Lett. B 669 (2008) 223 [arXiv:0807.4909 [hep-ex]].

[20] I. Caprini, J. Phys. A: Math. Gen. 14 (1981) 1271.

[21] I. Caprini, I. Guiasu and E. E. Radescu, Phys. Rev. D 25 (1982) 1808.

[22] G. Köpp, Phys. Rev. D 10 (1974) 932.

[23] R. Omnès, Nuovo Cim. 8 (1958) 316.

[24] R. García-Martín, R. Kamiński, J. R. Peláez, J. Ruiz de Elvira and F. J. Ynduráin, Phys. Rev. D 83 (2011) 074004 [arXiv:1102.2183 [hep-ph]].

[25] I. Caprini, G. Colangelo and H. Leutwyler, Eur. Phys. J. C 72 (2012) 1860 [arXiv:1111.7160 [hep-ph]]; in preparation.

[26] K. M. Watson, Phys. Rev. 95 (1954) 228.

[27] N. N. Khuri and S. B. Treiman, Phys. Rev. 119 (1960) 1115.

[28] K. A. Olive et al. [Particle Data Group Collaboration], Chin. Phys. C 38 (2014) 090001.

[29] I. Caprini, Eur. Phys. J. C 13 (2000) 471 [hep-ph/9907227].

[30] P. A. Baikov, K. G. Chetyrkin and J. H. Kühn, Phys. Rev. Lett. 101 (2008) 012002 [arXiv:0801.1821 [hep-ph]].

[31] M. Beneke and M. Jamin, JHEP 0809 (2008) 044 [arXiv:0806.3156 [hep-ph]].

[32] E. Braaten, S. Narison and A. Pich, Nucl. Phys. B 373 (1992) 581. 
[33] M. A. Shifman, A. I. Vainshtein and V. I. Zakharov, Nucl. Phys. B 147 (1979) 385; Nucl. Phys. B 147 (1979) 448.

[34] B. Aubert et al. [BaBar Collaboration], Phys. Rev. Lett. 103 (2009) 231801 [arXiv:0908.3589 [hep-ex]].

[35] B. Ananthanarayan, I. Caprini, D. Das and I. S. Imsong, Eur. Phys. J. C 72 (2012) 2192 [arXiv:1209.0379 [hep-ph]].

[36] B. Ananthanarayan, I. Caprini and I. S. Imsong, Phys. Rev. D 85 (2012) 096006 [arXiv:1203.5398 [hep-ph]].

[37] P. L. Duren, Theory of $H^{p}$ Spaces, Academic Press, New York (1970). 